\title{
Young stars in nearby early-type galaxies: SED fitting based on ultraviolet (UV) and optical imaging
}

\author{
Hyunjin Jeong ${ }^{1}$, Sukyoung K. Yi ${ }^{2}$, Martin Bureau ${ }^{3}$ \\ and Roger L. Davies ${ }^{3}$ \\ ${ }^{1}$ Korea Astronomy and Space Science Institute, Daejeon 305-348, Korea \\ ${ }^{2}$ Department of Astronomy, Yonsei University, Seoul 120-749, Korea \\ ${ }^{3}$ Sub-Department of Astrophysics, University of Oxford, Oxford OX1 3RH, UK
}

email: hyunjin@kasi.re.kr

\begin{abstract}
Recent studies from the Galaxy Evolution Explore (GALEX) ultraviolet (UV) data have demonstrated that the recent star formation is more common in early-type galaxies (ETGs) than we used to believe. The UV is one order of magnitude more sensitive than the optical to the presence of young stellar populations. The near-ultraviolet (NUV) lights of ETGs, especially, are used to reveal their residual star formation history. Here we used the GALEX UV data of 34 nearby early-type galaxies from the SAURON sample, all of which have optical data from MDM Observatory. At least $15 \%$ of the galaxies in this sample show blue UV-optical colours suggesting recent star formation (Jeong et al. 2009). These NUV blue galaxies are generally low velocity dispersion systems and change the slopes of scaling relations (colour-magnitude relations and fundamental planes) and increase the scatters. To quantify the amount of recent star formation in our sample, we assume two bursts of star formation, allowing us to constrain the age and mass fraction of the young component pixel by pixel (Jeong et al. 2007). The pixelby-pixel SED fitting based on UV and optical imaging reveals that the mass fraction of young $(<1$ Gyr old) stars in ETGs varies between 1 and $3 \%$ in the nearby universe (Jeong et al. in prep.). We will compare our results with the prediction from the hierarchical merger paradigm to understand the mechanism of low-level recent star formation observed in early-type galaxies.
\end{abstract}

Keywords. galaxies: elliptical and lenticular, cD - galaxies: evolution - galaxies: photometry - galaxies: structure - ultraviolet: galaxies.

\section{Introduction}

One of the greatest goals of modern astronomy is to understand how galaxies form and evolve. While a wealth of information is now available at medium and high redshifts, those studies rely on a detailed understanding of the local galaxy population, and much insight can be gained from studying the fossil record in nearby objects. To probe the mass assembly and star formation histories of galaxies in a hierarchical context, simultaneously constraining their dynamics and stellar population is the key. In particular, a thorough understanding of the star formation history of early-type galaxy promises key insights into the evolution of galaxies as a whole, and it strongly constrains galaxy formation models.

The exact formation mechanism of early-type galaxies remains one of the long-standing debates of modern astrophysics. Optical colour-magnitude relations (CMRs) of early-type galaxies consistently reveal a small scatter around the mean relation for the past several billion years (e.g. Bower, Lucey \& Ellis 1992), apparently supporting the classical collapse model. Similarly, it is well known that early-type galaxies occupy a two-dimensional plane 

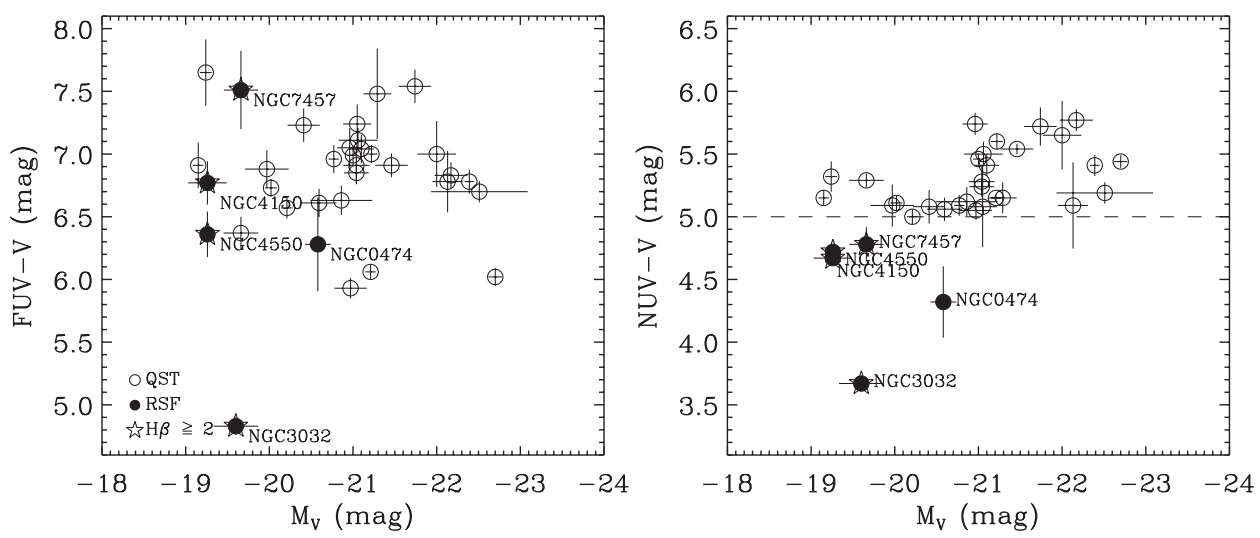

Figure 1. UV CMRs of our 34 SAURON early-type galaxies. The dashed line indicates the $\mathrm{NUV}-\mathrm{V}=5.0$ cut-off for recent star formation. Using this standard, we divided the sample into quiescent (QST; open symbols) early-type galaxies and recent star formation (RSF, filled symbols) galaxies.

in the three-dimensional manifold of their global structural parameters (effective radius, velocity dispersion and effective surface brightness) which is called the "fundamental plane (FP)" (e.g. Djorgovski \& Davis 1987, Dressler et al. 1987). The scatter in the fundamental plane was first thought to be small enough to represent further evidence for the monolithic scenario. However, the observed discrepancy or tilt of the fundamental plane with respect to the virial expectation has prompted many debates to explain its origin (see e.g. D'Onofrio et al. 2006 and references therein).

Recent advances in observational techniques are making the picture increasingly clear. The first is the advent of deep imaging facilities. Deep imaging surveys have shown that many of the "simple systems" do possess shells, tidal features and signatures of ongoing or recent star formation. The second advance is integral-field spectroscopy that makes it possible to obtain spatially resolved maps of various properties of galaxies. For example, the SAURON team (see e.g. Bacon et al. 2001, de Zeeuw et al. 2002) surveyed the two-dimensional stellar and ionised-gas kinematics and stellar populations of 72 nearby early-type galaxies and found a rich diversity in their dynamics, discovering numerous central disks and kinematically-decoupled cores. Such properties may be taken as evidence against the classical monolithic collapse model. Furthermore, the recent availability of survey data in the ultraviolet (e.g. GALEX) has reinvigorated the study of early-type galaxies and forced us to revisit assumptions about their evolution (see e.g. Yi et al. 2005).

\section{Observations and data reduction}

We observed 34 early-type galaxies from the SAURON project with the GALEX and the MDM Observatory 1.3-m McGraw-Hill Telescope. The GALEX UV images are delivered pre-processed but we undertook our own estimate of the sky values. And then, we carried out surface photometry by measuring the surface brightness along elliptical annuli after masking-out Sextractor-detected sources. From the radial surface brightness profiles, we derived total apparent magnitudes by extrapolating the growth curves to infinity. 



Figure 2. Fundamental Planes in the FUV and NUV bands. Symbols are the same as in Figure 1 and linear fits to the two subsamples (whole sample, quiescent galaxies only) are shown as dotted and solid lines, respectively.

\section{Results}

Numerous investigations can be performed with such a database. But, in this paper, we focus on the effects of star formation on the scaling relations. CMRs of early-type galaxies have been used as a tool for tracing their star formation histories. The NUV band is particularly sensitive to the presence of young stellar populations. The NUV CMR, therefore, is a good tool for tracking recent star formation. Figure 1 shows the UV-optical CMRs. The empirical demarcation of NUV-V $=5.0$ for identifying recent star formation galaxies is shown as a horizontal line in the NUV-V CMR. Using this empirical criterion, we have identified the galaxies that are likely to have experienced a recent star formation as filled symbols. The overall fraction of galaxies with recent star formation is $15 \%(5 / 34)$. We label galaxies with $\mathrm{NUV}-\mathrm{V} \geqslant 5.0$ as quiescent (QST) galaxies (open symbols). Furthermore, stars indicate Balmer absorption line strength $\mathrm{H} \beta \gtrsim 2 \AA$. Supporting our empirical threshold, four out of five recent star formation candidates also show an enhanced $\mathrm{H} \beta$ line strength. Our star formation interpretation is also consistent with the results of the molecular gas emission surveys. The CO detection rate decreases monotonically from recent star formation galaxies to quiescent galaxies. However, there is no clear dependence of $\mathrm{FUV}-\mathrm{V}$ on $\mathrm{M}_{V}$ in our sample.

The fundamental plane is another key scaling relation of early-type galaxies. It has long been recognised that the effective radius $\left(\mathrm{R}_{\mathrm{e}}\right)$, the mean effective surface brightness $\left(\langle\mu\rangle_{\mathrm{e}}\right)$ and the velocity dispersion $\left(\sigma_{\mathrm{e}}\right)$ are mutually correlated and unified in a two-dimensional manifold. Under the assumption of structural homology (i.e. all early-type galaxies have the same mass distribution and kinematics), the virial theorem predicts that the fundamental plane parameters should scale in a specific manner, but observations reveal a tilt away from the virial prediction. In Figure 2, we present the fundamental planes in the FUV and NUV bands, using the same symbols as in Figure 1. Recent star formation galaxies systematically deviate from the best-fit planes (dotted lines) so as to create shallower slopes, and they significantly increase the scatter. Considering our NUV fundamental plane fit for the entire sample, our slope is significantly different from the virial expectation. However, if we exclude recent star formation galaxies we recover almost all of the virial prediction. We thus conclude that a significant fraction of the fundamental plane tilt and scatter is due to low-mass early-type galaxies with young stellar populations.

In order to constrain the age and mass fraction of the young component pixel by pixel, we fit the observed colours (FUV-V and NUV-V) to simple two-population (young 

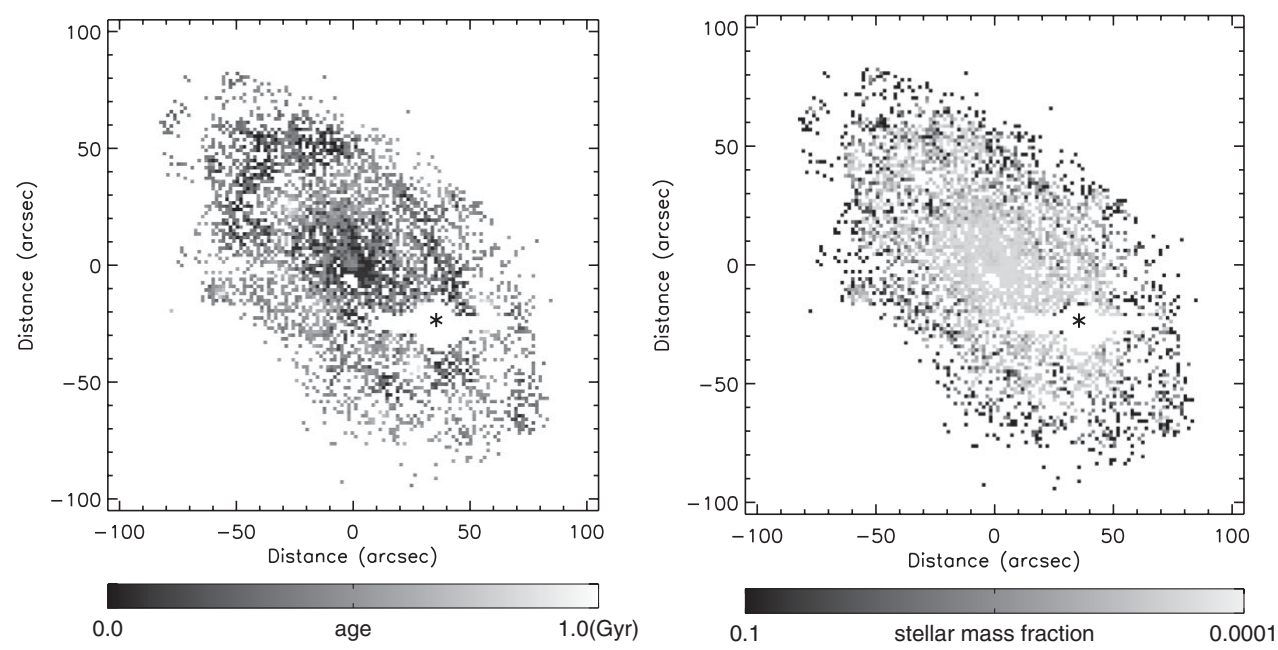

Figure 3. Two-component fit maps. A star masks the area contaminated by the foreground star.

and old) models from Yi (2003) and obtain a probability distribution of the age and mass fraction of the young stellar population. The UV data set is able to provide good constraints on the age and mass estimates of the young stellar populations in early-type galaxies. The inclusion of FUV data particulary yields a better fit to the model. The best estimates of one sample galaxy (NGC 2974) are shown in Figure 3. The age map shows very young stars $(<500 \mathrm{Myr})$ in both the central regions and in and around the UV rings. On the other hand, the mass fraction map shows that the fractional contribution of the young component increases outward. We calculate the total mass fraction of young stars in this galaxy to be around $1 \%$.

\section{Future works}

Star formation seems to have been more common in early-type galaxies than we used to believe. Early-type galaxies in the nearby universe have about $1-3 \%$ of their stellar mass in stars younger than 1 Gyr old. However, it is still unclear what is the main driver of star formation in early-type galaxies. We will compare our results with the prediction from the hierarchical merger paradigm and attempt to understand the overall assembly history of early-type galaxies.

\section{References}

Bacon, R. et al. 2001, MNRAS, 326, 23

Bower R. G., Lucey J. R., \& Ellis R. 1992, MNRAS, 254, 589

de Zeeuw, P. T. et al. 2002, MNRAS, 329, 513

Djorgovski, S. \& Davis, M. 1987, ApJ, 313, 59

D'Onofrio, M., Valentinuzzi, T., Secco, L., Caimmi, R., \& Bindoni, D. 2006, NewA Rev., 50, 447

Dressler, A., Lynden-Bell, D., Burstein, D., Davies, R. L., Faber, S. M., Terlevich, R. J., \& Vegner, G. 1987, ApJ, 313, 42

Jeong, H., Bureau, M., Yi, S. K., Krajnovi'c D., \& Davies, R. L. 2007, MNRAS, 376, 1021

Jeong, H., et al. 2009, MNRAS, 398, 2028

Yi, S. K. 2003, ApJ, 582, 202

Yi, S. K. et al. 2005, ApJ, 619, L111 